\title{
The IR gluon propagator from lattice QCD
}

\author{
Paulo J. Silva ${ }^{* \dagger}$ and Orlando Oliveira \\ Centro de Física Computacional, Departamento de Física, Universidade de Coimbra \\ 3004-516 Coimbra, Portugal. \\ E-mail: psilva@teor.fis.uc.pt, orlando@teor.fis.uc.pt
}

The gluon propagator is computed in large asymmetric lattices, accessing momenta around 100 $\mathrm{MeV}$ and smaller. Our study tries to check the compatibility of the recent solutions of the gluonghost Dyson-Schwinger equations with lattice results. In particular the exponent $\kappa$, which characterizes the solutions for the infrared, is measured. Results favours a vanishing zero momentum gluon propagator. We also report on the compatibility of various functional forms used to fit the Dyson-Schwinger solution for the full range of momenta and the lattice gluon propagator.

XXIIIrd International Symposium on Lattice Field Theory

25-30 July 2005

Trinity College, Dublin, Ireland

\footnotetext{
* Speaker.

${ }^{\dagger}$ Supported by FCT via grant SFRH/BD/10740/2002.
} 
Quantum chromodynamics (QCD) is the theory that describes the interaction between quarks and gluons. Two first principles approaches to non-perturbative QCD are Dyson-Schwinger equations (DSE) and the lattice formulation of QCD (LQCD). Solving the DSE, an infinite tower of equations, requires a truncation scheme and a parametrization of higher order vertices [1]. On the other hand, in LQCD one has to care about finite volume [2] and finite space effects. If handling finite space effects requires smaller lattice spacing, i.e. higher $\beta$ values, finite volume effects requires large volumes.

Recently, there has been some progress on the solution of the DSE. Assuming ghost dominance, the equations were solved in the infrared (IR) limit. The solution shows a vanishing zero momentum gluon propagator [3]. Moreover, the coupled gluon-ghost equations were solved numerically and analitical functions found that fitted the solution $[4,5]$. On the lattice, the gluon propagator has been revisited a number of times. However, due to the lattice sizes used in the investigations, the IR region was not yet properly accessed. The motivation of our study is to look at the deep IR gluon propagator and to try to check the compatibility between the two approaches, i.e. to test the IR DSE solution for the gluon propagator and to see if the same functional forms describe the lattice data and the DSE solution. In order to try to investigate the deep IR region, we consider large assymetric 4D lattices, i.e. lattices with a very large time extension [6].

\section{The lattice setup}

In this investigation we consider $\mathrm{SU}(3)$ pure gauge, Wilson action at $\beta=6.0$. The value of the lattice spacing to convert lattice into physical units being $a^{-1}=1.943 \mathrm{GeV}$ [7]. For the lattices $16^{3} \times 128$ and $16^{3} \times 256$, the gauge configurations ${ }^{1}$ were generated as described in [6]. For the smallest (largest) lattice 164 (155) configurations were generated. The large time extension allows to access momenta of about $95 \mathrm{MeV}$ for the smallest lattice and $48 \mathrm{MeV}$ for the largest lattice. For the definition of the gluon propagator, notation and the gauge fixing method see [8].

\section{Gluon dressing function}

In figure 1 the bare gluon dressing function is shown for all pure temporal and pure spatial momenta. The data shows volume effects, with the spatial momenta giving larger values for the dressing function. The data suggests that one should not mix temporal and spatial momenta.

For the IR study, we will consider only pure temporal momenta. For these momenta and the two lattices, the gluon dressing function and the propagator are reported in figure 2. The two data sets are compatible within errors. Note that this does not mean that finite volume effects are negligable [2].

\subsection{IR gluon dressing function}

The IR analytical solution of the DSE for the gluon dressing function being $Z\left(q^{2}\right) \sim\left(q^{2}\right)^{2 \kappa}$, we first try to fit the lattice data to this expression ${ }^{2}$. An acceptable $\chi^{2} /$ d.o.f. $=0.40$ was obtained

\footnotetext{
${ }^{1}$ All confi gurations were generated with MILC code http: //physics. indiana. edu/ sg/milc. html.

${ }^{2}$ The fi ts were done separately for each lattice. The statistical errors on all fi tting parameters were estimated using 1600 bootstrap samples.
} 

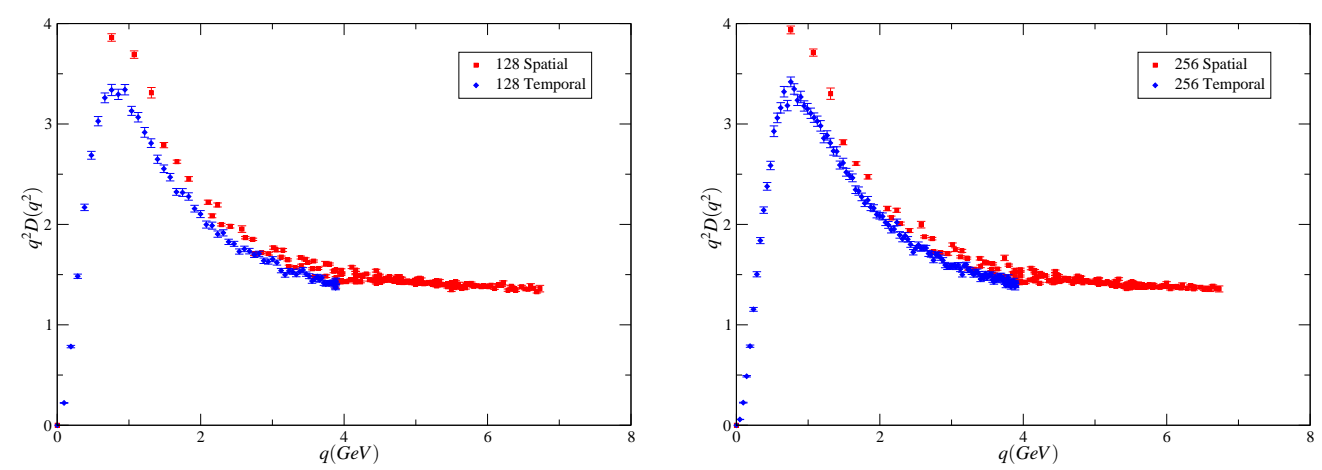

Figure 1: Gluon dressing function for the smaller (left) and larger (right) lattices.
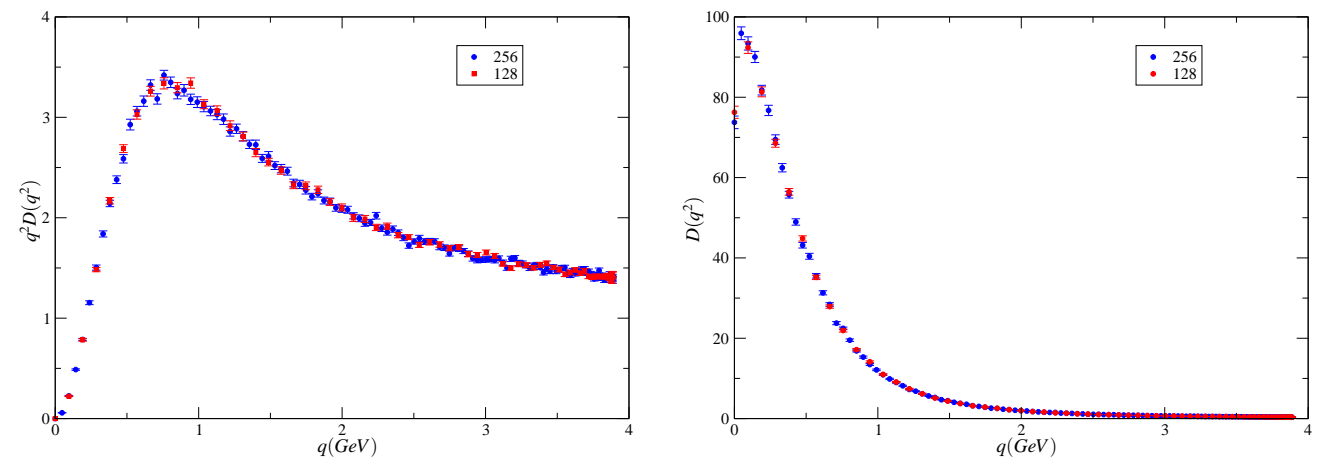

Figure 2: Gluon dressing function (left) and propagator (right) for pure temporal momenta.

only by fitting the first three lowest momenta (excluding the zero momentum) for the largest lattice $(|q| \leq 143 \mathrm{MeV})$, meaning that the DSE solution seems to be valid only for momenta below 150 $\mathrm{MeV}$. The measured $\kappa=0.4859_{-24}^{+22}$ does not support a vanishing zero momentum gluon propagator. In order to test the validity of the result, corrections to the DSE solution were considered, fitting the smallest possible range of momenta. The results are in table 1.

\begin{tabular}{|lccc|}
\hline $16^{3} \times 256$ & $\boldsymbol{q}_{\max }$ & $\boldsymbol{\kappa}$ & $\chi^{2} /$ d.o.f. \\
\hline$\omega\left(q^{2}\right)^{2 \kappa}\left(1+a q^{2}\right)$ & $191 \mathrm{MeV}$ & $0.5070_{-50}^{+36}$ & 0.44 \\
$\omega\left(q^{2}\right)^{2 \kappa}\left(1+a q^{2}+b q^{4}\right)$ & $238 \mathrm{MeV}$ & $0.5131_{-64}^{+67}$ & 1.03 \\
$\omega\left(q^{2}\right)^{2 \kappa}\left(1+a q^{2}+b q^{4}+c q^{6}\right)$ & $286 \mathrm{MeV}$ & $0.5146_{-96}^{+70}$ & 1.36 \\
\hline
\end{tabular}

Table 1: Fits to polinomial corrections to the DSE analytical solution.

It is interesting to observe that, with corrections to the analitycal DSE solution, $\kappa$ becomes larger than 0.5. Moreover, the results reported in table 1 are compatible within errors and give an error weighted mean value of $\kappa=0.5108(13)$. Note that the $\kappa$ just reported are not compatible with the direct fit to the DSE solution. 
In [4] the DSE solution was well described by the two expressions

$$
Z_{c u t}\left(q^{2}\right)=\omega\left(\frac{q^{2}}{\Lambda_{Q C D}^{2}+q^{2}}\right)^{2 \kappa} \quad, Z_{\text {pole }}\left(q^{2}\right)=\omega \frac{\left(q^{2}\right)^{2 \kappa}}{\left(\Lambda_{Q C D}^{2}\right)^{2 \kappa}+\left(q^{2}\right)^{2 \kappa}},
$$

which associate, respectively, a cut and a pole to the propagator in the IR region. The lattice data is well described by both expressions with no clear preference for the cut or the pole. The results of the fits, for the momenta region starting at the lowest nonzero momentum up to $q_{\max }$, are reported in table 2. Note that all fits have good $\chi^{2} /$ d.o.f. and, for each function, the fitted parameters are compatible within one standard deviation.

\begin{tabular}{|cccccc|}
\hline & Lattice & $q_{\max }$ & $\kappa$ & $\Lambda_{Q C D}(\mathrm{MeV})$ & $\chi^{2} /$ d.o.f. \\
\hline$Z_{\text {cut }}$ & $16^{3} \times 256$ & $664 \mathrm{MeV}$ & $0.5090_{-20}^{+19}$ & $409_{-4}^{+4}$ & 0.71 \\
& $16^{3} \times 128$ & $570 \mathrm{MeV}$ & $0.5117_{-46}^{+48}$ & $417_{-8}^{+8}$ & 1.25 \\
\hline$Z_{\text {pole }}$ & $16^{3} \times 256$ & $664 \mathrm{MeV}$ & $0.5077_{-17}^{+16}$ & $409_{-3}^{+4}$ & 0.69 \\
& $16^{3} \times 128$ & $570 \mathrm{MeV}$ & $0.5100_{-31}^{+38}$ & $416_{-8}^{+6}$ & 1.15 \\
\hline
\end{tabular}

Table 2: $Z_{c u t}$ and $Z_{\text {pole }}$ fits.

\subsection{UV gluon dressing function}

As in previous studies $[9,8]$ we fit the UV data to the one-loop perturbative gluon dressing function,

$$
\omega\left[\frac{1}{2} \ln \left(\frac{q^{2}}{\Lambda^{2}}\right)\right]^{-\gamma}
$$

where $\gamma=-13 / 22$ is the gluon anomalous dimension. In the UV region two types of momenta were considered, namely pure temporal and cylinder plus conic cuts as defined in [9]. The results can be seen in table 3 for the largest acceptable momentum range. They show that the UV region is well described by the one-loop perturbative result, with the temporal momenta following the perturbative behaviour earlier than the cylindrical+conical cut momenta. Note that since the fits are to the bare data, the fitted $\Lambda$ 's should not be compared directly with standard values.

\begin{tabular}{|c|cc|cc|}
\hline Lattice & \multicolumn{2}{|c|}{ Temporal } & \multicolumn{2}{c|}{ Cuts } \\
& $1.8 G e V \leq\|q\| \leq 3.9 \mathrm{GeV}$ & $2.8 \mathrm{GeV} \leq\|q\| \leq 7.8 \mathrm{GeV}$ \\
& $\Lambda$ & $\boldsymbol{\chi}^{2} / \boldsymbol{d} . \boldsymbol{o . f}$. & $\boldsymbol{\Lambda}$ & $\boldsymbol{\chi}^{\mathbf{2}} / \boldsymbol{d} . \boldsymbol{o . f}$. \\
\hline $16^{3} \times 128$ & $1006_{-9}^{+7} \mathrm{MeV}$ & 1.2 & $640_{-5}^{+5} \mathrm{MeV}$ & 1.1 \\
$16^{3} \times 256$ & $990_{-6}^{+6} \mathrm{MeV}$ & 1.1 & $643_{-4}^{+4} \mathrm{MeV}$ & 1.2 \\
\hline
\end{tabular}

Table 3: UV fits.

\subsection{The gluon dressing function}

In what concerns the full data set, it was tested against the following formulas $Z_{f i t}\left(q^{2}\right)=$ $B\left(q^{2}\right)\left(\alpha_{f i t}\left(q^{2}\right)\right)^{-\gamma}$, with $B\left(q^{2}\right)=Z_{\text {pole }, \text { cut }}\left(q^{2}\right)$ and using two different definitions for the running 
coupling,

$$
\begin{aligned}
\alpha_{P}\left(q^{2}\right) & =\frac{1}{1+\frac{q^{2}}{\Lambda_{Q C D}^{2}}}\left[\alpha(0)+\frac{q^{2}}{\Lambda_{Q C D}^{2}} \times \frac{4 \pi}{\beta_{0}}\left(\frac{1}{\ln \left(q^{2} / \Lambda_{Q C D}^{2}\right)}-\frac{1}{q^{2} / \Lambda_{Q C D}^{2}-1}\right)\right], \beta_{0}=11, \\
\alpha_{L N}\left(q^{2}\right) & =\frac{\alpha(0)}{\ln \left[e+a_{1}\left(q^{2} / \Lambda_{Q C D}^{2}\right)^{a_{2}}\right]}
\end{aligned}
$$

proposed in [4,5]. The fits with $\alpha_{P}\left(q^{2}\right)$ had always $\chi^{2} /$ d.o.f. $\geq 2$, i.e. the lattice data is not described by such a running coupling constant. The lattice data adjusts better to $\alpha_{L N}\left(q^{2}\right)$ as can be seen by the results reported in table 4 . Note that the $\kappa$ values are now larger and closer to the DSE figures, than those measured in the IR region.

\begin{tabular}{|lccccc|}
\hline $\boldsymbol{B}=\boldsymbol{Z}_{\text {cut }}$ & $\boldsymbol{\kappa}$ & $\boldsymbol{\Lambda}_{Q C D}(\mathbf{M e V})$ & $\boldsymbol{a}_{\mathbf{1}}$ & $\boldsymbol{a}_{\mathbf{2}}$ & $\boldsymbol{\chi}^{\mathbf{2}} / \boldsymbol{d} . \boldsymbol{o . f}$. \\
\hline $16^{3} \times 128$ & $0.5435_{-41}^{+36}$ & $364_{-4}^{+4}$ & $0.0062_{-3}^{+3}$ & $2.44_{-1}^{+2}$ & 1.82 \\
$16^{3} \times 256$ & $0.5244_{-15}^{+21}$ & $374_{-2}^{+2}$ & $0.0072_{-3}^{+3}$ & $2.424_{-11}^{+10}$ & 1.73 \\
\hline \hline $\boldsymbol{B}=\boldsymbol{Z}_{\text {pole }}$ & $\boldsymbol{\kappa}$ & $\boldsymbol{\Lambda}_{Q C D}(\mathbf{M e V})$ & $\boldsymbol{a}_{\mathbf{1}}$ & $\boldsymbol{a}_{\mathbf{2}}$ & $\boldsymbol{\chi}^{\mathbf{2}} / \boldsymbol{d} . \boldsymbol{o} \boldsymbol{f}$. \\
\hline $16^{3} \times 128$ & $0.5335_{-26}^{+23}$ & $373_{-3}^{+3}$ & $0.0081_{-3}^{+3}$ & $2.36_{-2}^{+2}$ & 1.65 \\
$16^{3} \times 256$ & $0.5217_{-14}^{+14}$ & $377_{-2}^{+2}$ & $0.0082_{-3}^{+3}$ & $2.36_{-1}^{+1}$ & 1.61 \\
\hline
\end{tabular}

Table 4: Fits to all lattice data.

The value of the zero momentum running coupling can be computed from the asymptotic behaviour of the QCD $\beta$-function and from $\alpha_{L N}\left(p^{2}\right), \alpha(0)=\left(4 \pi / \beta_{0}\right) a_{2}$. Note that the values reported in table 5 are not too far from the DSE numbers.

\begin{tabular}{|ccc|}
\hline $\boldsymbol{\alpha}(\mathbf{0})$ & $Z_{\text {cut }}$ & $Z_{\text {pole }}$ \\
\hline $16^{3} \times 128$ & $2.79_{-1}^{+2}$ & $2.70_{-2}^{+2}$ \\
$16^{3} \times 256$ & $2.77_{-1}^{+1}$ & $2.70_{-1}^{+1}$ \\
\hline
\end{tabular}

Table 5: Values of $\alpha(0)$ extracted from $\alpha_{L N}\left(p^{2}\right)$.

\section{Gribov copies}

For the smallest lattice, we look for possible Gribov copies effects comparing the method used in [6] with that described in [10]. The ratio of the propagators computed from the two methods, $D_{I D} / D_{C E A S D}$, for pure temporal momenta is shown in figure 3. Although we have a ratio that, for many momenta, is not compatible with one, we cannot conclude in favour of any systematic effect $^{3}$. This means either that our statistics is too small to resolve Gribov copies or they do not play a significant role for such lattices.

\footnotetext{
${ }^{3}$ In [8] the effect of the Gribov copies was estimated as a 2 to $3 \sigma$ effect.
} 


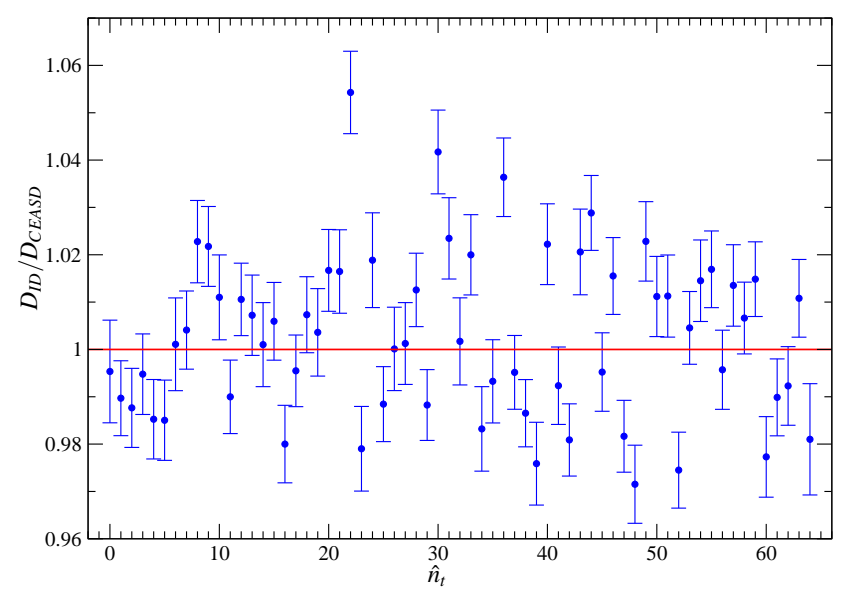

Figure 3: Gribov copy effects in $16^{3} \times 128$ configurations.

\section{Conclusions}

The lattice data seems to be compatible with functional forms that describe the gluon-ghost DSE numerical solution. In what concerns the vanishing of the zero momentum gluon propagator, our data does not provide a clear answer but it favours a vanishing propagator in agreement with the DSE solution, flow equations studies [11, 12] and time independent stochastic quantisation [13].

\section{References}

[1] See, for example, R. Alkofer, L. von Smekal, Phys. Rept. 353 (2001) 281 [hep-ph/ 0007355$].$

[2] For an exploratory study of the finite volume effects on IR gluon propagator see O. Oliveira, P. J. Silva, "Finite volume effects in the gluon propagator", these proceedings [hep-lat/0509037].

[3] C. Lerche, L. von Smekal, Phys. Rev. D65 (2002) 125006 [hep-ph/ 0202194 ].

[4] R. Alkofer, W. Detmold, C. S. Fischer, P. Maris, Phys. Rev. D70 (2004) 014014 [hep-ph/0309077].

[5] C. S. Fischer, R. Alkofer, Phys. Rev. D67 (2003) 094020 [hep-ph/ 0301094 ].

[6] O. Oliveira, P. J. Silva, AIP Conf. Proc. 756 (2005) 290 [hep-lat / 0410048 ].

[7] G. S. Bali, K. Schilling, Phys. Rev. D47 (1993) 661 [hep-lat/9208028].

[8] P. J. Silva, O. Oliveira, Nucl. Phys. B690 (2004) 177 [hep-lat / 0403026 ].

[9] D. B. Leinweber et al Phys. Rev. D60 (1999) 094507; erratum-ibid Phys. Rev. D61 (2000) 079901 [hep-lat/9811027].

[10] O. Oliveira, P. J. Silva, Comp. Phys. Comm. 158 (2004) 73 [hep-lat/030 9184].

[11] J. M. Pawlowski, D. F. Litim, S. Nedelko, L. von Smekal, Phys. Rev. Lett. 93 (2004) 152002.

[12] C. S. Fischer, H. Gies, JHEP 0410 (2004) 048 [hep-ph / 040808 9].

[13] D. Zwanziger, Phys. Rev. D67 (2003) 105001 [hep-th/0206053]. 